\title{
PATRONES HISTÓRICOS DE CUEREO EN LOBOS MARINOS: LA EVIDENCIA DE LOS ABRIGOS 1 Y 2 DE PLAYA YÁMANA (ISLA LIVINGSTON, ANTÁRTICA CHILENA)
}

\author{
ISABEL CARTAJENA F." Y RAFAEL LABARCA E.
}

\begin{abstract}
RESUMEN
Se presentan los resultados del análisis de huellas de corte en restos óseos de pinnípedos provenientes de dos yacimientos loberos históricos, localizados en cabo Shirreff, isla Livingston, Antártica Chilena. Se describe la ubicación, disposición y características morfológicas de las huellas, con el objeto de establecer los modos de procesamiento llevados a cabo en los yacimientos estudiados. Los resultados indican que los cortes fueron hechos con instrumentos metálicos, en su mayoría orientados a la extracción de la piel, con un procedimiento muy estandarizado.

PALABRAS CLAVES: Huellas de corte, patrón de faenamiento, pinnípedos, Antártica.

\section{HISTORIC AGE SKINNING PATTERNS OF SEA WOLVES: THE ARCHAEOFAUNISTIC RECORD OF ABRIGOS 1 AND 2, YÁMANA BEACH (LIVINGSTON ISLAND, CHILEAN ANTARCTICA)}

ABSTRACT

The paper presents the results of an analysis of cut marks on pinniped bones recovered at two historic age sites located in Cape Shirreff, Livingston Islands, Chilean Antarctica. The location, disposition and morphological characteristics of each mark are described in order to identify butchering patterns employed at the studied sites. The study concludes that the marks were made with a metal tool and generally followed a very standardized procedure geared towards the skinning of animals.
\end{abstract}

KEY WORDS: Cut marks, butchering pattern, pinnipeds, Antarctica.

* Departamento de Antropología. Universidad de Chile. Ignacio Carrera Pinto 1045. E-mail: icartaje@uchile.cl Juan Moya Morales \#910, Ñuñoa. E-mail: r.labarca.e@gmail.com 


\section{INTRODUCCIÓN}

Durante los siglos XVIII, XIX y comienzos del XX distintas flotas europeas y norteamericanas desarrollaron en las costas de Chile una caza sistemática e indiscriminada de diversas especies de mamíferos marinos (focas, lobos de mar y ballenas principalmente) con el objeto de extraer pieles, aceites y carnes, bienes muy cotizados en los mercados internacionales (Pereira Salas 1971; Martinic 1987; Zarankin y Senatore 1999). Esta situación se tradujo en un paulatino agotamiento de los cotos de caza ubicados en sectores septentrionales (p. ej. archipiélago Juan Fernández, isla grande de Chiloé, cabo de Hornos), lo que forzó gradualmente a los loberos a buscar nuevas áreas de caza cada vez más al sur. En el contexto de esta carrera impuesta por la demanda creciente de bienes, en 1819 fueron descubiertas las islas Shetland del Sur, aparentemente por la nave norteamericana Hersilia (Pinochet de la Barra 1976; Zarankin y Senatore 1999; Stehberg 2003). La importante colonia de pinnípedos que residía en el sector motivó la llegada de diversas flotas de loberos, los que explotaron de manera intermitente, por casi un siglo, los recursos pelágicos del lugar y de otras islas e islotes adyacentes, desplegando campamentos y utilizando reparos rocosos en sectores cercanos a la costa para el procesamiento de los animales cazados (Stehberg 2003).

Las investigaciones arqueológicas realizadas en la Antártica septentrional, específicamente en el archipiélago de las Shetland del Sur, han confirmado la información histórica, ya que han sido reconocidos y estudiados diversos asentamientos de loberos (Stehberg y Cabeza 1987; Stehberg y Lucero 1996; Zarankin y Senatore 1999; Stehberg 2003). Particularmente los trabajos de Stehberg en territorio chileno han permitido el registro de cerca de 12 asentamientos históricos, recuperándose una importante cantidad de material artefactual y ecofactual (Stehberg 2003). En estos trabajos, además, fueron recobrados instrumentos de clara manufactura indígena, confirmando la presencia de mano de obra local en las labores de faenamiento de los mamíferos marinos (Stehberg y Blanco 2002).

Pese a que el principal objetivo de los cazadores extranjeros era la obtención de pieles de lobo de mar, los datos históricos disponibles son insuficientes para caracterizar el modo de procesamiento de los animales (Pereira Salas 1971; Stehberg 2003). Una forma de subsanar esta falencia, es a través del estudio detallado de las modificaciones culturales presentes en los restos de pinnípedos recuperados de contextos loberos. Puesto que se trata de una explotación sistemática, recurrente en el tiempo y con un objetivo específico, se planteó como hipótesis inicial la existencia de un modo particular de procesamiento de la carcasa (Yellen 1991), altamente eficiente, orientado a la optimización de tiempo, energía y materiales. Este patrón sería visible en el registro arqueológico a través de la recurrencia en la ubicación y disposición de las huellas de corte en las unidades anatómicas registradas, las que debían ser coherentes, en primer término, con el descuerado del animal y, en menor medida, con el descarne y la desarticulación de las extremidades.

Con el objetivo de contrastar esta hipótesis, el presente artículo entrega los resultados de los análisis arqueofaunísticos efectuados a dos conjuntos óseos de pinnípedos recuperados en playa Yámana, isla Livingston, Shetland del Sur. Se describen en detalle las distintas marcas detectadas y se discute la posibilidad de establecer patrones de faenamiento a partir de la evidencia registrada.

\section{ANTECEDENTES: LAS EXCAVACIONES EN PLAYA YÁMANA}

Playa Yámana $\left(62^{\circ} 27^{\prime} \mathrm{S} 60^{\circ} 47^{\prime} \mathrm{W}\right)$ se ubica en la costa oeste del cabo Shirreff, en la isla Livingston, la que forma parte del archipiélago de las Shetland del Sur (Fig. 1). De acuerdo a Stehberg presenta forma triangular con un largo de $270 \mathrm{~m}$ y un ancho máximo de $70 \mathrm{~m}$ (...) hacia su extremo oriente presenta afloramientos rocosos aislados de entre 3 y $10 \mathrm{~m}$ de largo y socavados por la acción marina formando oquedades naturales (Stehberg 2003:86-87). Un total de seis reparos rocosos fueron excavados estratigráficamente, recuperándose material cultural exclusivamente en los Abrigos 1 y 2. En ambos aleros se intervino un total de $6 \mathrm{~m}^{2}$ a través de dos cuadrículas de $1 \times 3 \mathrm{~m}$, excavadas con niveles artificiales de $5 \mathrm{~cm}$ hasta una profundidad máxima de $60 \mathrm{~cm}$. La evidencia obtenida se condice plenamente con un campamento histórico, ya que se compone principalmente de cerámica vidriada roja (contenedores de gin de origen inglés), fragmentos de loza azul, botellas de vidrio, clavos y otros 


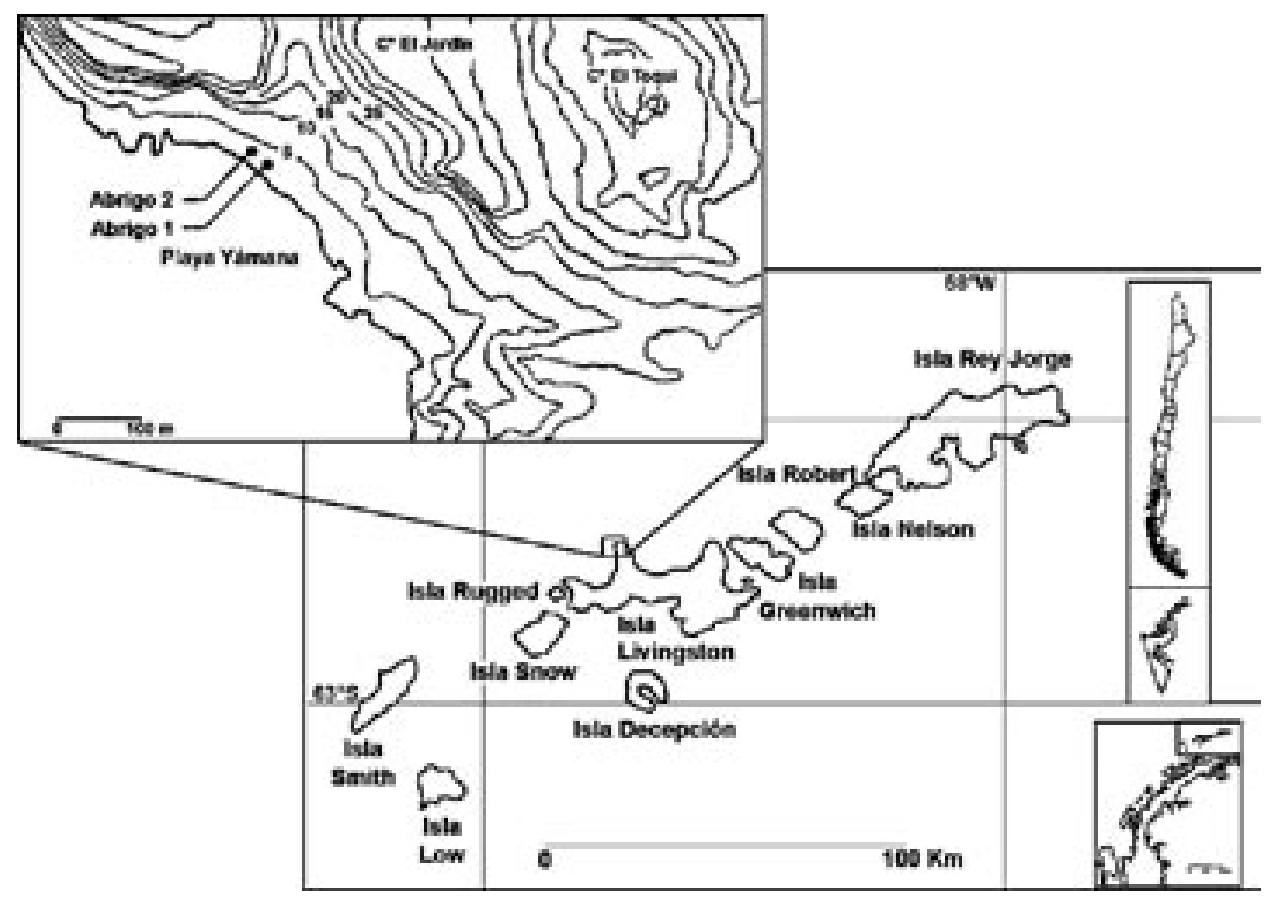

Fig. 1.

fragmentos de metal, partes de zapatos, corchos, maderas de especies provenientes del hemisferio norte y abundantes restos óseos de pinnípedos. No se apreciaron diferencias estratigráficas por lo que no se pudo segregar eventos ocupacionales. No obstante lo anterior, es muy probable que la ocupación dé cuenta de las actividades realizadas entre 1819 y 1924 , ya que corresponde al momento en que la isla fue visitada de manera más intensiva (Stehberg 2003).

\section{MATERIAL Y MÉTODO}

Los materiales provienen íntegramente de los Abrigos 1 y 2 de playa Yámana, ubicados en el cabo Shirreff, isla Livingston, en Territorio Antártico Chileno. La muestra se compone de 1399 especímenes óseos (NISP Abrigo 1=154; NISP Abrigo 2=1132 y NISP sin referencia $=113$ ), correspondientes a 14 kg aproximadamente.

Para la determinación anatómica y taxonómica se utilizaron criterios morfológicos a través de esqueletos de pinnípedos recolectados en el sector y guías osteológicas (p.e. Gilbert 1973; King 1964; Schiavini 1992).
Para medir la integridad del conjunto se consideró los criterios de meteorización propuestos por Beherensmeyer (1978), puesto que la conservación del material incidirá directamente en la posibilidad de preservar las marcas presentes en la superficie de los huesos. Debido a la ubicación de los yacimientos, no se advirtieron huellas de carácter natural (marcas de carroñeros, raíces, roedores), por lo que el análisis se centró en las huellas culturales, particularmente aquellas de corte. En este sentido, la identificación de una huella de corte se basa en dos criterios fundamentales: por un lado la morfología de ésta, tanto macroscópica como microscópica, y por otro, las características contextuales (Bunn 1981; Potts y Shipman 1981; Mengoni 1988 a y b; Lyman 1994; Fisher 1995). Debido a que se trata de un sitio de loberos históricos, se hizo especial hincapié en la morfología de las huellas dejadas por artefactos metálicos, siguiendo los criterios de Walker y Long (1977), Gifford-González (1974) y Greenfield (1999). Éstas se caracterizan por presentar una sección transversal en $\mathrm{V}$ con lados que convergen en la parte inferior, sin microestriaciones internas. La sección transversal podrá presentarse simétrica o asimétrica dependiendo del ángulo con 
que se utilizó el artefacto. En general, son estrechas, superficiales y no siguen el contorno del hueso, sin embargo, el ancho y profundidad dependerá del largo de la hoja del agente productor. Si bien, la utilización de SEM (Scanning electron microscope) o de imágenes digitales permite un mayor refinamiento metodológico para diferenciar entre huellas producidas por artefactos líticos o metálicos (Gilbert y Richards 2000), se ha sugerido que las marcas producidas por cuchillos metálicos pueden ser reconocidas fácilmente a través de criterios morfológicos utilizando técnicas de aumento de bajo poder (Blumenschine et al. 1996).

En relación al análisis contextual de las huellas, en términos generales, un patrón de marcas humanas se caracterizará por su recurrencia y por tener una ubicación compatible con la anatomía económica del animal, situación que fue posible cotejar mediante criterios posicionales y distribucionales. Para tal efecto, y mediante un análisis macroscópico, se tomaron en cuenta: a) ubicación; b) orientación; c) frecuencia; d) distribución; e) longitud; f) profundidad y g) ancho (Mengoni 1988b, 1999).

Con el fin de correlacionar las huellas de corte con actividades específicas se consideraron:

a) Cuereo: las marcas se ubicarán donde sea más operativo desollar el animal, generalmente en las extremidades inferiores beneficiando las partes de acuerdo al uso que se les dará.

b) Desarticulación: las marcas se encontrarán en los lugares de inserción muscular y tendones cerca de las articulaciones.

c) Despostamiento y consumo: las marcas se acomodarán de acuerdo a los requerimientos alimenticios del grupo (consumo de carne, grasa, etc.), en general donde el rendimiento cárneo sea mayor (Binford 1981).

Mediante una lupa estereoscópica NIKON (SM-10, Tipo 102) con ocular de $15 x$ y objetivo de 0.66 a 4x, se analizó la sección transversal de las huellas con el fin de determinar rasgos específicos asociados al agente productor. Se escogieron para ser fotografiadas unidades óseas representativas de los distintos tipos de huellas definidas, en la medida de que la localización de las marcas y estado de conservación del hueso permitieran su registro. Las piezas completas fueron fotografiadas por la cara que presentaba marcas; con posterioridad, cada huella fue fotografiada con un aumento de $25 \mathrm{x}$, usando una cámara Canon AE-1 adosada a una lupa binocular Motic e iluminada con luz externa desde diversos ángulos (ca. 700 wats).

\section{RESULTADOS}

Los huesos presentan blanqueo, escamación y agrietamiento de las piezas en general y deterioro de huesos planos. Si bien el color indica que muchos de los ejemplares estuvieron expuestos, gran parte del conjunto presenta estados de meteorización bajos y un tono amarillento que indica enterramiento donde las condiciones de meteorización son más lentas. La determinación taxonómica de los restos se realizó teniendo en consideración la morfología del paladar, la mandíbula y los caninos, los que presentan rasgos propios de Arctocephalus sp. (Schiavini 1992:1314), probablemente A. gazella bien documentado para la Antártica.

Considerando ambos abrigos, se estimó un total de 31 individuos a partir del fémur izquierdo (Stehberg 2003, Tabla 9). No obstante, el MNI con marcas alcanza a nueve, calculado a partir de la ulna izquierda, el que aumenta a 11 si se agregan dos radios izquierdos sin fusionar. En general, el número de unidades con huellas es bajo, ya que del total del conjunto, sólo el 4,07\% (NISP=56) presenta este atributo. Esta baja frecuencia no indica necesariamente que algunos animales no habrían sido faenados o bien faenados parcialmente, ya que las marcas de corte corresponden a un epifenómeno, es decir, un accidente durante el proceso de reducción de las carcasas, por lo que su presencia y frecuencia dependerá de diversas variables, como el tipo de presa, la morfología del hueso, instrumental empleado, intensidad del procesamiento, condición de la carcasa, pericia de faenador, tiempo destinado al faenamiento, entre muchos otros (Haynes 1991; Lyman 1992; De Nigris 1999; Lupo y O' Connell 2002; Egeland 2003; Lyman 2005; Dewbury y Russell 2007).

La Tabla 1 muestra la distribución de las marcas de acuerdo a las unidades anatómicas registradas (Fig. 2), en donde los porcentajes de restos con huellas de corte fueron reescalados en un índice de base cien con el fin de apreciar las diferencias relativas entre las diferentes unidades anatómicas que presentan marcas. Aun cuando el porcentaje de unidades con modificaciones es bajo, se puede apreciar que las 
Tabla 1. Distribución de huellas de corte por unidad anatómica (\%) en restos de pinnípedos de Playa Yámana.

\begin{tabular}{lcccc}
\hline \multicolumn{1}{c}{ Unidad anatómica } & MNE & MNE c/ Huellas & \%MNE c/ Huellas & Índice de huellas \\
\hline Costillas & 450 & 9 & 2 & 8,7 \\
Mandíbula & 66 & 2 & 3 & 13,0 \\
Cráneo & 50 & 2 & 4 & 17,4 \\
Húmero & 60 & 3 & 5 & 21,7 \\
Escápula & 33 & 3 & 9 & 39,1 \\
Ulna & 68 & 13 & 19 & 82,6 \\
Radio & 62 & 13 & 21 & 91,3 \\
Tibia & 48 & 11 & 23 & 100,0 \\
\hline
\end{tabular}

huellas se concentran en las extremidades distales, específicamente radio, ulna y tibia, en desmedro de las unidades proximales (húmero y fémur) y del esqueleto axial. Esta situación no se encuentra mediada por la frecuencia de partes esqueletarias, ya que el número de restos con huellas de corte para cada una de las unidades anatómicas, no se encuentra correlacionado con la abundancia de $(\rho=0.267 \mathrm{p}=0.52)$.

En general, la ubicación y orientación de las modificaciones en estos especímenes presentan una relativa homogeneidad, ya que se concentran en el sector medial o distal de la diáfisis y se disponen de forma más o menos horizontal en relación al eje del hueso. Particularmente en el radio las marcas se agrupan en el sector medio de la diáfisis, en su cara lateral, principalmente orientadas hacia el borde palmar (Fig. 2, huellas 1, 2 y 3; Fig. 3 a, b). En todos los casos las marcas se ubican de manera relativamente perpendicular a la orientación de la pieza, y presentan largos que oscilan entre los $3 y$ $11 \mathrm{~mm}$. En sólo un caso se aprecian dos huellas asociadas, lo que indica que se trata de movimientos poco reiterativos. A nivel macroscópico, las marcas exhiben una sección muy regular, en forma de $\mathrm{V} y$ con paredes rectas (Tabla 2).

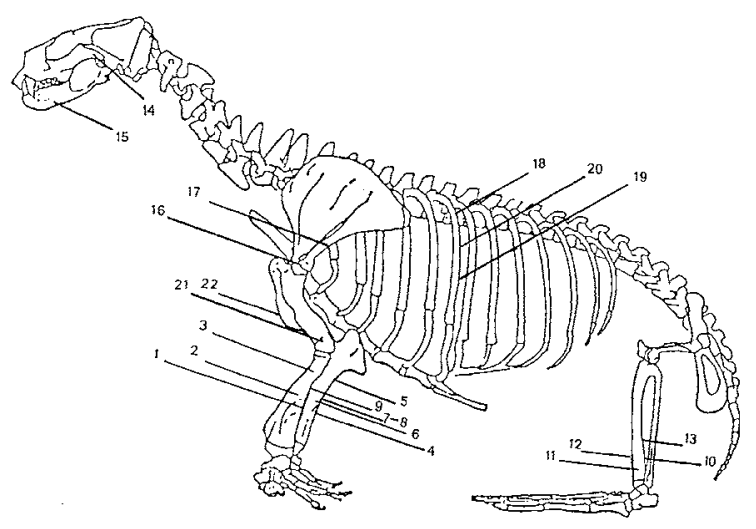

Fig. 2.

Al igual que lo observado en el radio, las marcas en la ulna se ubican principalmente en el sector medial de la diáfisis (12 casos), registrándose sólo una unidad con marcas en el sector proximal. Son particularmente abundantes aquellas registradas en la cara palmar (6 casos), aun cuando se identificaron huellas en las caras lateral, medial y dorsal (Fig. 2, huellas 4, 5, 6 y 7; Fig. 3c y d; Tabla 3). En todos los casos las huellas se disponen de manera oblicua $\mathrm{u}$ horizontal en relación al eje del hueso. El rango

Tabla 2. Resumen de características de las huellas de corte en radios de Playa Yámana.

\begin{tabular}{|c|c|c|c|c|c|c|}
\hline \multicolumn{7}{|c|}{ Radio } \\
\hline Descripción & MNE & $\begin{array}{l}\text { Frecuencia } \\
\text { huella }\end{array}$ & Disposición & $\begin{array}{l}\text { Longitud } \\
(\mathrm{mm})\end{array}$ & Profundidad & $\begin{array}{c}\text { Actividad } \\
\text { Relacionada }\end{array}$ \\
\hline $\begin{array}{l}\text { Diáfisis media, cara lateral hacia } \\
\text { borde palmar }\end{array}$ & 1 & 2 & $\begin{array}{l}\text { Transversal- } \\
\text { subparalelas }\end{array}$ & $5-3$ & Superficial & Cuereo \\
\hline $\begin{array}{l}\text { Diáfisis media, cara lateral hacia } \\
\text { borde palmar }\end{array}$ & 5 & 1 & Transversal & $4 / 3 / 4 / 4 / 7$ & Superficial & Cuereo \\
\hline $\begin{array}{l}\text { Diáfisis media, cara lateral hacia } \\
\text { borde palmar }\end{array}$ & 1 & 1 & Oblicua & 4 & Superficial & Cuereo \\
\hline Diáfisis media, cara lateral & 3 & 1 & Oblicua & $10 / 11 / 5$ & Superficial & Cuereo \\
\hline Diáfisis media, cara lateral & 1 & 1 & Transversal & 5 & Superficial & Cuereo \\
\hline Diáfisis proximal, cara lateral & 2 & 1 & Oblicua & $4 / 3$ & Superficial & Cuereo \\
\hline
\end{tabular}


Tabla 3. Resumen de características de las huellas de corte en ulnas de Playa Yámana.

\begin{tabular}{|c|c|c|c|c|c|c|}
\hline \multicolumn{7}{|c|}{ Ulna } \\
\hline Descripción & MNE & $\begin{array}{l}\text { Frecuencia } \\
\text { huella }\end{array}$ & Disposición & $\begin{array}{l}\text { Longitud } \\
(\mathrm{mm})\end{array}$ & Profundidad & $\begin{array}{c}\text { Actividad } \\
\text { Relacionada } \\
\end{array}$ \\
\hline Diáfisis proximal, cara palmar & 1 & 1 & Transversal & 3 & Superficial & Cuereo \\
\hline Diáfisis media, cara dorsal & 1 & 2 & $\begin{array}{l}\text { Transversal - } \\
\text { subparalelas }\end{array}$ & $6-6$ & Superficial & Cuereo \\
\hline $\begin{array}{l}\text { Diáfisis media, cara lateral hacia } \\
\text { borde palmar }\end{array}$ & 2 & 1 & Transversal & $1 / 2$ & Superficial & Cuereo \\
\hline $\begin{array}{l}\text { Diáfisis media, cara medial hacia el } \\
\text { borde dorsal }\end{array}$ & 2 & 1 & Transversal & $5 / 1$ & Superficial & Cuereo \\
\hline $\begin{array}{l}\text { Diáfisis media, cara medial hacia } \\
\text { borde palmar }\end{array}$ & 1 & $1 / 2$ & $\begin{array}{c}\text { Oblicua/ } \\
\text { Oblicua-subparalelas }\end{array}$ & $4 / 2-3$ & Superficial & $\begin{array}{l}\text { Cuereo- } \\
\text { Consumo }\end{array}$ \\
\hline Diáfisis media, cara palmar & 5 & 1 & Transversal & $4 / 4 / 4 / 3 / 3$ & Superficial & Cuereo \\
\hline Diáfisis media, cara palmar & 1 & 2 & $\begin{array}{c}\text { Transversal / } \\
\text { paralelas }\end{array}$ & $1 / 1$ & Superficial & Cuereo \\
\hline
\end{tabular}

de longitud comprende marcas entre 1 y $6 \mathrm{~mm}$, siendo en promedio más cortas que las del radio. En este sentido, las marcas más cortas coinciden con la ubicación en los bordes. Nuevamente se registra principalmente sólo una huella por unidad.

En la tibia fueron identificadas huellas en dos sectores: diáfisis media y diáfisis distal. Las marcas, no obstante, se concentran en este último sector (11 casos), particularmente en la cara dorsal, tanto

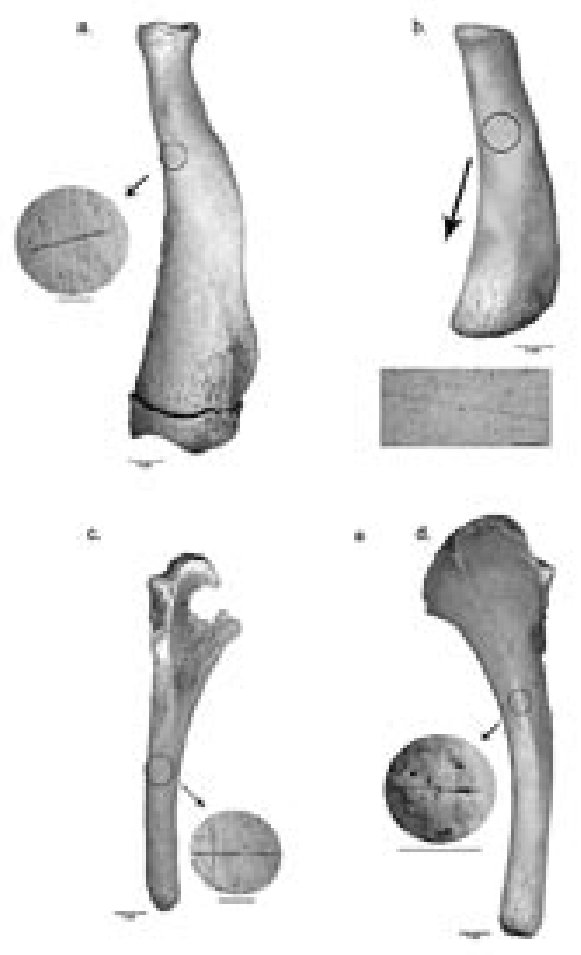

Fig. 3. hacia el borde medial como lateral (Fig. 2, huellas 10, 11, 12 y 13; Fig. 4a y b; Tabla 4). No se registra ninguna marca en la cara plantar. Las longitudes, al igual que las profundidades y las frecuencias, son un poco más variables, aun cuando predominan las huellas de corte con orientación oblicua al eje del hueso, de sección en $\mathrm{V}$ y paredes rectas.

Por su parte, en el cráneo se aprecian huellas exclusivamente en el arco cigomático, en su cara exterior hacia el borde inferior, a la altura de la unión con el cóndilo mandibular. Las huellas se dan en agrupaciones paralelas y son un poco más profundas (Fig. 2, huella 14; Fig. 5a; Tabla 5). Son igualmente escasas las huellas en la mandíbula, las que se disponen en la cara exterior hacia el borde inferior, en grupos paralelos con orientación oblicua (Fig. 2, huella 15; Fig. 5b, Tabla 6). Para ambos casos se aprecian mayores frecuencias de cortes, indicando
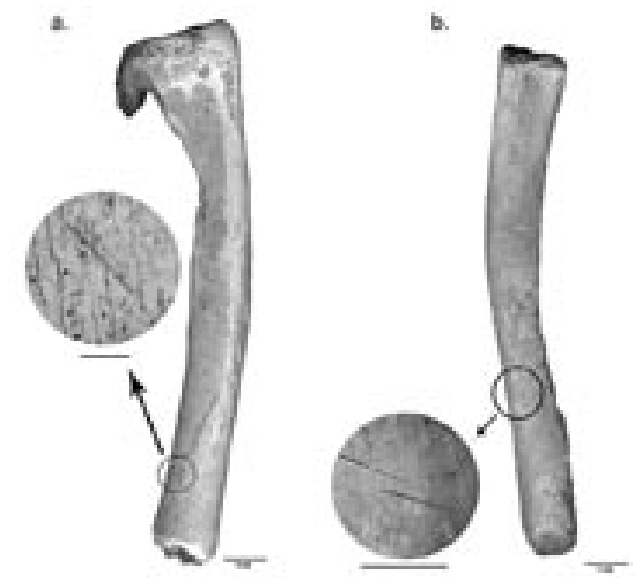

Fig. 4. 
Tabla 4. Resumen de características de las huellas de corte de tibias de Playa Yámana.

\begin{tabular}{|c|c|c|c|c|c|c|}
\hline \multicolumn{7}{|c|}{ Tibia } \\
\hline Descripción & MNE & $\begin{array}{c}\text { Frecuencia } \\
\text { huella }\end{array}$ & Disposición & $\begin{array}{c}\text { Longitud } \\
(\mathrm{mm})\end{array}$ & Profundidad & $\begin{array}{c}\text { Actividad } \\
\text { Relacionada } \\
\end{array}$ \\
\hline $\begin{array}{l}\text { Diáfisis distal, cara dorsal/ } \\
\text { diáfisis media, cara dorsal }\end{array}$ & 1 & $3 / 1$ & $\begin{array}{c}\text { Oblicua-subparalelas/ } \\
\text { Oblicua }\end{array}$ & $3-3-4 / 3$ & Superficial & Cuereo \\
\hline Diáfisis distal, cara dorsal & 1 & 2 & $\begin{array}{l}\text { Transversal - } \\
\text { Paralelas }\end{array}$ & $2-9$ & Superficial & Cuereo \\
\hline Diáfisis distal, cara dorsal & 1 & 2 & $\begin{array}{l}\text { Oblicua/ } \\
\text { Subparalelas }\end{array}$ & $2-4$ & Superficial & Cuereo \\
\hline $\begin{array}{l}\text { Diáfisis distal, cara dorsal } \\
\text { hacia el borde medial }\end{array}$ & 1 & 1 & Transversal & 4 & Superficial & Cuereo \\
\hline $\begin{array}{l}\text { Diáfisis distal, cara dorsal } \\
\text { hacia el borde medial }\end{array}$ & 1 & 1 & Oblicua & 3 & Superficial & Cuereo \\
\hline $\begin{array}{l}\text { Diáfisis distal, cara dorsal } \\
\text { hacia el borde lateral }\end{array}$ & 1 & 3 & $\begin{array}{l}\text { Transversal / } \\
\text { Subparalelas }\end{array}$ & $8-5-3$ & Media & Cuereo \\
\hline $\begin{array}{l}\text { Diáfisis distal, cara dorsal } \\
\text { hacia el borde lateral }\end{array}$ & 1 & 2 & $\begin{array}{l}\text { Oblicua- } \\
\text { Subparalelas }\end{array}$ & 3-2 & Superficial & Cuereo \\
\hline Diáfisis distal, cara medial & 1 & 1 & Oblicua & 6 & Superficial & Cuereo \\
\hline Diáfisis distal, cara lateral & 1 & 1 & Oblicua & 4 & Superficial & Cuereo \\
\hline Diáfisis distal, cara lateral & 2 & 1 & Oblicua & 8 & Media & Cuereo \\
\hline
\end{tabular}

Tabla 5. Resumen de características de las huellas de corte en cráneos de Playa Yámana.

\begin{tabular}{lcccccc}
\hline \multicolumn{1}{c}{ Descripción } & MNE & $\begin{array}{c}\text { Frecuencia } \\
\text { huella }\end{array}$ & Disposición & $\begin{array}{c}\text { Longitud } \\
(\mathrm{mm})\end{array}$ & Profundidad & Actividad Relacionada \\
\hline $\begin{array}{l}\text { Cara exterior del } \\
\text { arco cigomático }\end{array}$ & 1 & 1 & Oblicua & 7 & Superficial & $\begin{array}{c}\text { Desarticulación/ } \\
\text { Cuereo } \\
\begin{array}{l}\text { Borde inferior del } \\
\text { arco cigomático }\end{array}\end{array}$ \\
\hline
\end{tabular}

Tabla 6. Resumen de características de las huellas de corte en mandíbulas de Playa Yámana.

\begin{tabular}{ccccccc}
\hline & \multicolumn{5}{c}{ Mandíbula } \\
\hline \multicolumn{1}{c}{ Descripción } & MNE & $\begin{array}{c}\text { Frecuencia } \\
\text { huella }\end{array}$ & Disposición & $\begin{array}{c}\text { Longitud c/u } \\
\text { (mm) }\end{array}$ & Profundidad & Actividad Relacionada \\
\hline $\begin{array}{l}\text { Cara exterior hacia } \\
\text { el borde inferior }\end{array}$ & 2 & 2 & $\begin{array}{c}\text { Oblicua- } \\
\text { paralelas }\end{array}$ & $3-2 / 8-4$ & $\begin{array}{c}\text { Media/ } \\
\text { Superficial }\end{array}$ & Cuereo \\
\hline
\end{tabular}

Tabla 7. Resumen de características de las huellas de corte en escápulas de Playa Yámana.

\begin{tabular}{lcccccc}
\hline \multicolumn{1}{c}{ Descripción } & MNE & $\begin{array}{c}\text { Frecuencia } \\
\text { huella }\end{array}$ & Disposición & $\begin{array}{c}\text { Longitud } \\
\text { (mm) }\end{array}$ & Profundidad & Actividad Relacionada \\
\hline $\begin{array}{l}\text { Cara ventral cerca del borde } \\
\text { posterior }\end{array}$ & 1 & 3 & $\begin{array}{c}\text { Oblicua- } \\
\text { paralelas }\end{array}$ & $7-10-15$ & Profunda & $?$ \\
Cara ventral & 1 & 2 & Oblicua & $10-15$ & Profunda & $?$ \\
Cavidad glenoidea & 1 & 1 & Oblicua & 4 & Superficial & Desarticulación \\
\hline
\end{tabular}

una mayor reiteración en el proceso de faenamiento, el que podría estar relacionado con el cuereo.

En la escápula se observan marcas en dos sectores: cavidad glenoidea (Fig. 2, huella 16) y cara ventral hacia borde caudal NISP $=2$ (Fig. 2, huella 17; Tabla 7). Una de las marcas, ubicada en la cavidad glenoidea sigue el mismo patrón uniforme de las descritas anteriormente y seguramente está en relación con la desarticulación del húmero. Sin embargo, existen dos ejemplares que presentan huellas muy anchas y largas. La sección tiende más bien a ser en "U", con estrías paralelas al eje longitudinal del corte y con una de las paredes rectas. No pudieron ser asociadas a ninguna actividad en particular ni a un agente productor. 
Tabla 8. Resumen de características de las huellas de corte de húmeros de Playa Yámana.

\begin{tabular}{lccccccc}
\hline \multicolumn{1}{c}{ Descripción } & MNE & MNI & $\begin{array}{c}\text { Frecuencia } \\
\text { huella }\end{array}$ & Disposición & $\begin{array}{c}\text { Longitud } \\
\text { (mm) }\end{array}$ & Profundidad & $\begin{array}{c}\text { Actividad } \\
\text { Relacionada }\end{array}$ \\
\hline $\begin{array}{l}\text { Diáfisis distal, cara lateral } \\
\text { hacia epicóndilo lateral }\end{array}$ & 1 & 1 & 2 & $\begin{array}{c}\text { Oblicua- } \\
\text { paralelas }\end{array}$ & $2-2$ & Media & Desarticulación \\
$\begin{array}{l}\text { Diáfisis distal, cara caudal } \\
\text { hacia epicóndilo medial }\end{array}$ & 1 & 1 & 1 & Oblicua & 4 & Superficial & Desarticulación \\
Diáfisis distal, cara lateral & 1 & 1 & 2 & $\begin{array}{c}\text { Oblicua- } \\
\text { paralelas }\end{array}$ & $7-3$ & Profunda & $?$ \\
\hline
\end{tabular}

En el húmero se identificaron marcas sólo en la diáfisis distal, tanto hacia la cara lateral, como medial (Fig. 2, huellas 21 y 22; Tabla 8). Dos especímenes presentan marcas oblicuas de mediana profundidad en la diáfisis distal que seguramente guardan relación con la desarticulación de la ulna proximal. Uno de los huesos presenta una marca muy similar a las de las escápulas: larga, profunda y ancha. Tampoco se pudo determinar el agente productor ni una actividad específica.

Finalmente, en las costillas se aprecian marcas en nueve casos, concentrados principalmente en la cara medial (Fig. 2, huellas 18, 19 y 20; Fig. 5c; Tabla 9). A diferencia de lo observado en otras unidades anatómicas, en este caso las marcas se distribuyen en grupos paralelos y subparalelos de a dos o más, particularmente en la cara interna, indicando una acción reiterativa. Las longitudes son variables, pero en un mismo grupo presentan un largo similar, predominando las con orientación oblicuas. Su presencia podría estar relacionada con labores de extracción de carne.

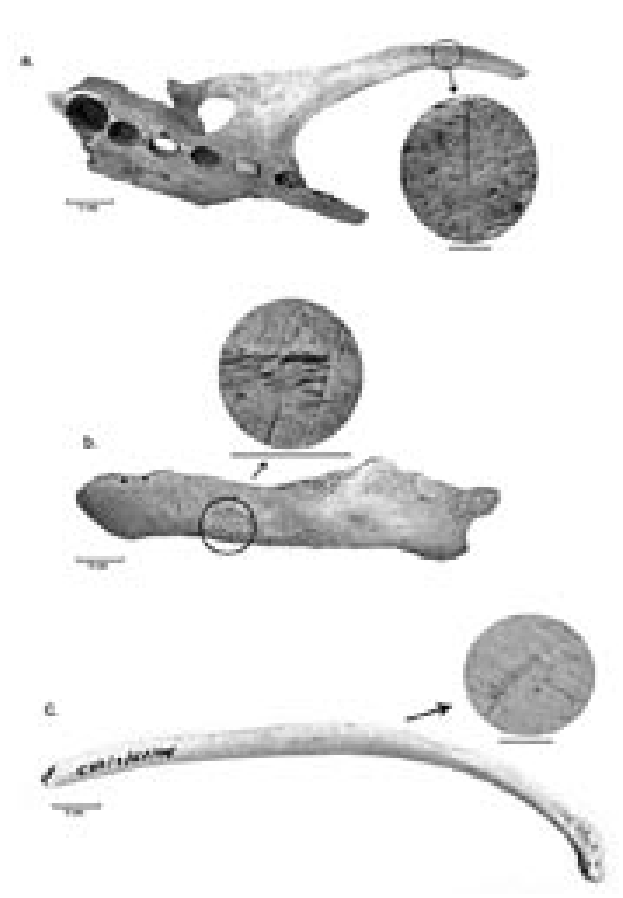

Fig. 5.

Tabla 9. Resumen de características de las huellas de corte en costillas de Playa Yámana.

\begin{tabular}{|c|c|c|c|c|c|c|c|}
\hline & & & & Costilla & & & \\
\hline Descripción & MNE & MNI & $\begin{array}{c}\text { Frecuencia } \\
\text { huella }\end{array}$ & Disposición & $\begin{array}{l}\text { Longitud } \\
(\mathrm{mm})\end{array}$ & Profundidad & $\begin{array}{c}\text { Actividad } \\
\text { Relacionada }\end{array}$ \\
\hline Cara medial & 1 & 1 & 1 & Transversal & 5 & Superficial & Carneo \\
\hline $\begin{array}{l}\text { Cara medial, } \\
\text { porción dorsal }\end{array}$ & 2 & 1 & 2 & $\begin{array}{c}\text { Oblicua- } \\
\text { subparalelas }\end{array}$ & $2-3 / 2-3$ & Superficial & Carneo \\
\hline $\begin{array}{l}\text { Cara medial, } \\
\text { porción media }\end{array}$ & 1 & 1 & 4 & $\begin{array}{l}\text { Oblicua- } \\
\text { paralelas }\end{array}$ & $4-4-3-2$ & Superficial & Carneo \\
\hline $\begin{array}{l}\text { Cara medial, } \\
\text { porción media }\end{array}$ & 2 & 1 & 2 & $\begin{array}{l}\text { Oblicua- } \\
\text { paralelas }\end{array}$ & $4-4 / 3-3$ & Superficial & Carneo \\
\hline $\begin{array}{l}\text { Cara medial, hacia } \\
\text { borde craneal }\end{array}$ & 1 & 1 & 2 & $\begin{array}{c}\text { Transversal - } \\
\text { paralelas }\end{array}$ & $3 / 1$ & Superficial & Carneo \\
\hline Cara lateral & 1 & 1 & 1 & Oblicua & 4 & Superficial & Carneo \\
\hline Borde caudal & 1 & 1 & 2 & $\begin{array}{c}\text { Oblicua- } \\
\text { subparalelas }\end{array}$ & $1 / 3$ & Media & $?$ \\
\hline
\end{tabular}




\section{DISCUSIÓN Y CONCLUSIONES}

En general, durante el cuereo hay pocas partes del artefacto que entran en contacto con el hueso, y por tanto sólo algunas partes esqueletarias presentarán marcas. La información etnográfica y arqueológica sugiere que las huellas de corte relacionadas con la extracción de la piel se concentran en las extremidades distales, tanto delanteras (radio-ulna distal y metacarpos) como traseras (tibia distal y metatarso proximal) y en la cabeza (Binford 1981; Wilson 1982; Lyman 1994; Fisher 1995). De igual forma, los resultados obtenidos por Lyman et al. (1992) en fócidos, sugieren que las marcas de cuereo se concentran en el radio y ulna medial para el caso del miembro anterior, mientras que en las extremidades posteriores se ubicarían cercanas a la articulación de los huesos cortos y cráneo (Lyman et al. 1992, Figure 1). En Playa Yámana, tanto las unidades anatómicas que presentan huellas de corte como la ubicación de éstas son compatibles con lo planteado anteriormente, por lo que se puede postular que en ambos abrigos rocosos se desarrollaron mayoritariamente actividades de cuereo o extracción de la piel, situación que permite confirmar la hipótesis inicial propuesta.

En relación a las extremidades proximales, el análisis conjunto de las huellas registradas en el radio y la ulna permite suponer que el proceso de extracción de la piel se realizaba mediante un corte circular o semicircular en el sector medio de estos huesos. En efecto, para el caso del radio, las marcas se concentran en la cara lateral, mientras que la ulna se registran marcas fundamentalmente en las caras medial, palmar y dorsal. La variación en la frecuencia de las huellas identificadas en los distintos sectores se encontraría mediada aparentemente por la morfología de las unidades óseas, ya que por ejemplo, la porción medial de la ulna es cóncava, en contraposición a la porción lateral del radio. En el caso de la tibia, las huellas de corte se concentran principalmente en las superficies dorsal, medial y en menor medida, lateral. La ubicación de las marcas indica que el cuereo se realizaría a partir de un corte similar al observado para las extremidades superiores, el que comenzaría en la cara lateral o medial de la tibia, atravesando la cara dorsal. De igual forma, las huellas ubicadas al lado exterior de la mandíbula también podrían estar asociadas a labores de cuereo.
Aquellas modificaciones ubicadas en los arcos cigomáticos podrían guardar relación con actividades de cuereo o bien con eventos de desarticulación para separar la mandíbula del cráneo a nivel de los cóndilos mandibulares. Otros indicios de desarticulación son las huellas presentes en la diáfisis distal de los húmeros, asociadas a la separación de la ulna proximal. Del mismo modo, la marca en la cavidad glenoidea de la escápula está relacionada con la desarticulación del húmero proximal. Éste se disloca fácilmente por lo que las huellas quedan generalmente en la articulación de la escápula (Lyman et al. 1992).

En menor medida, se advierten también huellas de corte producto de actividades de despostamiento, sobre todo en las costillas. Cabe recordar que esta unidad anatómica es la que presenta mayor rendimiento de carne (Lyman 1994). Al descuerar un lobo, cuero y grasa se mantienen unidos, quedando la carne adherida al cuerpo (Quiroz 1992). Las marcas características del despostamiento se presentan en grupos, paralelas y oblicuas, ajustándose las huellas de corte registradas a dicho patrón, aunque su frecuencia no se encuentra directamente relacionada con la cantidad de carne adherida a ésta (Pobiner y Braun 2005). La presencia de este tipo de marcas indica que los animales no sólo eran desollados sino que, además, habrían sido en parte consumidos, sugiriendo una utilización integral de este recurso. Esta información se condice con los antecedentes históricos, los que mencionan la carne de lobo de mar como una de las comidas a bordo de los navíos loberos (Pereira Salas 1971).

Mediante el análisis de la sección transversal de las huellas, éstas pudieron ser correlacionadas con el agente productor, tratándose en este caso de una hoja de metal (cuchillo metálico) (Walker y Long 1977; Greenfield 1999). Para el caso de las huellas en la escápula y húmero, cuya sección se presenta más redondeada, podría corresponder a un cuchillo con el borde embotado (Greenfield 1999), aun cuando no se descarta la posibilidad de que se trate de instrumentos líticos. La utilización de un artefacto metálico también puede ser corroborada por la información etnográfica, donde se describe el "cuchillo lobero": vamos corriendo cuchillo, el lobo no se parte, se pela.....ve que el lobo se pela redondo (Quiroz 1992:13). 
Las huellas de corte presentan en su mayoría una sección en $\mathrm{V}$ simétrica, lo que sugeriría que fueron producidas por un cuchillo metálico aplicado en un ángulo recto a la superficie del hueso. La uniformidad de las marcas sugiere que fueron generadas por un mismo artefacto o muy similar, puesto que con la variación de la hoja también varía el ancho de la huella, a excepción de los ya mencionados especímenes que presentan huellas muy anchas. La profundidad de la marca, por su parte, dependerá del accidente topográfico que entra en contacto con el artefacto cortante y de la fuerza que se ejerza. En nuestro caso de estudio, aparentemente se habría ejercido mayor fuerza al procesar las extremidades delanteras.

Una gran cantidad de factores determina la posibilidad de que el procesamiento de un determinado animal genere trazas visibles en los restos óseos (Lyman 1987, 1994; Fisher 1995). A esta situación se deben sumar los factores postepositacionales, que muchas veces ocultan, eliminan o simulan huellas de carácter cultural (Lyman 1994), por lo que se ha discutido enormemente la factibilidad de generar "patrones de faenamiento" a partir exclusivamente del "resultado del proceso de faenamiento" (sensu Lyman 1987). No obstante, la muestra analizada presenta diversos atributos particulares, los que considerados en conjunto, permitieron postular la existencia de un modo particular de procesamiento de lobos marinos orientado casi exclusivamente a la extracción de su piel: (1) contextos monocomponentes, cronológicamente acotados, funcionalmente similares y generados por una misma población; (2) ausencia de modificaciones naturales en los restos óseos; (3) marcada recurrencia en las unidades que presentan huellas de corte y, (4) marcada recurrencia en la ubicación, disposición y morfología de las marcas.

De acuerdo a Stehberg (Stehberg y Blanco 2002; Stehberg 2003), se ha recuperado evidencia empírica que permite demostrar la presencia de indígenas involucrados en la explotación de lobos marinos en el sector, por lo que resulta factible postular que éstos hayan sido los verdaderos encargados de la captura y procesamiento de los animales, debido a su acabado conocimiento de la fauna costera. A pesar de que este modo de procesamiento podría tener una data prehispánica, situación que podría contrastarse con el estudio de yacimientos arqueológicos prehistóricos del extremo austral que presenten restos de pinnípedos, es necesario considerar que éste también corresponde a un patrón general de cuereo como se observa en otras partes del mundo (Lyman et al. 1992).

\section{AGRADECIMIENTOS}

A Rubén Stehberg por brindarnos la posibilidad de revisar los materiales de Playa Yámana.

\section{BIBLIOGRAFÍA}

BEHERENSMEYER, A. 1978. Taphonomic and Ecologic Information from Bone Weathering. Paleobiology 4(2):150-162.

BINFORD, L. 1981. Bones: Ancient men modern myths. Academic Press, New York.

BLUMENSCHINE, R. J., C. W. MAREAN y S. D. CAPALDO 1996. Blind test of inter-analyst correspondence and accuracy in the identification of cut marks, percusion marks, and carnivore tooth marks on bone surfaces. Journal of Archaeological Science 23(4):493-507.

BUNN, H., 1981. Archaeological evidence for meat eating by Plio-Pleistocene hominids from Koobi Fora and Olduvai Gorge. Nature 291:575-577.

DE NIGRIS, M. 1999. Lo crudo y lo cocido: sobre los efectos de la cocción en la modificación ósea. Arqueología 9:239-264.

DEWBURY, A. G. y N. RUSSELL. 2007. Relative frequency of butchering cutmarks produced by obsidian and flint: an experimental approach. Journal of Archaeological Science 34:354-357.

EGELAND, C.P. 2003. Processing intensity and cutmark creation: an experimental approach. Plains Anthropologist 48:39-51

FISHER, J.W. 1995. Bone surface modifications in zooarchaeology. Journal of Archaeological Method and Theory 2:7-68.

GIFFORD-GONZÁLEZ, D. 1974. Ethnography Analogues for Interpreting Modified bones: Some Cases from East Africa. En: Bone Modification. Editado por R. Bonnichsen y M.H. Sorg, pp:179-246. University of Maine. Center for the Study of the First Americans, Orono.

GILBERT, M. 1973. Mammalian Osteoarchaeology: North America. Special publications, D. Evans. Ed. Missouri Archaeological Society.

GILBERT, W. H. y G. D. RICHARDS. 2000. Digital imaging of bone and tooth modification. The Anatomical Record (New Anat.) 261:237-246. 
GREENFIELD, H. 1999. The Origins of Metallurgy: Distinguishing Stone from Metal Cut-marks on bones from archaeological sites. Journal of Archaeological Science 26:797-808.

HAYNES, G. 1991. Mammoths, mastodonts, and elephants: Biology, behaviour, and the fossil record: Cambridge, United Kingdom, Cambridge University Press.

KING, J. 1964. Seals of the World. British Museum (Natural History). London.

LUPO, K D. y J. F. O'CONNELL. 2002. Cut and Tooth Mark Distributions on Large Animal Bones: Ethnoarchaeological Data from Hadza and Their Implications for Current Ideas about Early Human Carnivory. Journal of Archaeological Science. 29:85-109.

LYMAN, R. L. 1987. Archaeofaunas and Butchery Studies: A Taphonomic Perspective. En Advances in Archaeological Method and Theory 10. Editado por Michael B. Schiffer, pp. 249-337. Academic Press. San Diego.

LYMAN, R. L. 1992. Prehistoric Seal and Sea-Lion Butchering on the Southern Northwest Coast. American Antiquity 57(2):246-261

LYMAN, R. L. 1994. Vertebrate Taphonomy. Cambridge University Press.

LYMAN, R. L. 2005. Analyzing cut marks: lessons from artiodactyl remains in the northwestern United States. Journal of Archaeological Science 32: 1722-1732.

LYMAN, R., J. M. SAVELLE y P. WHITRIDGE 1992. Derivation and application of Meat Utility index for phocid seals. Journal of Archaeological Science 19: 531-555.

MARTINIC, M. 1987. Navegantes norteamericanos en Magallanes durante la primera mitad del Siglo XIX. Anales del Instituto de la Patagonia, Serie Ciencias Humanas 17:1-17.

MENGONI, G. 1988a. Análisis de materiales faunísticos de sitios arqueológicos. Xama 1:71-120. Mendoza, Argentina.

MENGONI, G. 1988b. El estudio de huellas en arqueofauna, una vía para reconstruir situaciones interactivas en contextos arqueológicos: aspectos teóricos y técnicas de análisis. En De Procesos, Contextos y Otros Huesos. Editado por N. Ratto y A. Haber, pp:17-27. ICA, UBA, Buenos Aires, Argentina.

MENGONI, G. 1999. Cazadores de Guanacos de la estepa Patagónica. Colección tesis doctorales. Ediciones de la Sociedad Argentina de Antropología.

PEREIRA SALAS, E 1971. Los primeros contactos entre Chile y Estados Unidos 1778-1809. Historia de las relaciones internacionales de Chile. Editorial Andrés Bello.
PINOCHET DE LA BARRA, O. 1976. La Antártida Chilena. Editorial Andrés Bello.

POBINER, B. L. y D. R. BRAUN. 2005. Strengthening the Inferential Link Between Cutmark Frequency Data and Oldowan Hominid Behavior: Results From Modern Butchery Experiments. Journal of Taphonomy 3(2-3):18-26

POTTS, R. y P. SHIPMAN. 1981. Cutmarks made by stone tools on bones from Olduvai Gorge, Tanzania. Nature 291:577-580.

QUIROZ, D. 1992. Lanza con lobos. Revista Museos 14:1214.

SCHIAVINI, A. 1992. Los pinnípedos del sitio Túnel I (canal Beagle, Tierra del Fuego, Argentina): el análisis faunístico. Cuadernos, Serie Técnica 2: 56 pp. Instituto Nacional de Antropología, Buenos Aires.

STEHBERG, R. 2003. Arqueología histórica antártica. Aborígenes sudamericanos en los mares subantárticos en el siglo XIX. Colección de Antropología No. VI. Dirección de Bibliotecas, Archivos y Museos, Centro de Investigaciones Diego Barros Arana.

STEHBERG, R. y A. CABEZA. 1987. Comienzos de la arqueología histórica antártica en el sitio Cuatro Pircas. Revista Chilena de Antropología 6:83-111.

STEHBERG, R. y V. LUCERO. 1996. Excavaciones arqueológicas en playa Yámana, cabo Shirreff, isla Livingston, Shetland del Sur, Antártica. Serie Científica INACH 46:59-81

STEHBERG, R y J. F. BLANCO. 2002. ¿Artefactos indígenas o chisperos para fusil? Un problema arqueológico en Isla Desolación, Antártica Chilena. Anales del Instituto de la Patagonia, Serie Ciencias Humanas 30:175-182.

WALKER, P. y J. LONG. 1977. An experimental study of the morphological characteristics of tool marks. American Antiquity 42(4):605-616.

WILSON, M. C. 1982. Cut marks and early Hominids: Evidence for skinning. Nature 298:303.

YELLEN, J. E. 1991. Small mammals: Kung San utilization and the production of faunal assemblages. Journal of Anthropological Archaeology 10:1-26.

ZARANKIN, A. y M. X. SENATORE 1999. Ocupación humana en tierras antárticas. Una aproximación arqueológica. En: Soplando el Viento. Actas de las III Jornadas de Arqueología de la Patagonia:629-644. 
